\title{
Least Square Approximations and Linear Values of Cooperative Games
}

\author{
Ulrich FAIGLE* $\quad$ Michel GRABISCH ${ }^{\dagger}$
}

March 29, 2018

\begin{abstract}
Many important values for cooperative games are known to arise from least square optimization problems. The present investigation develops an optimization framework to explain and clarify this phenomenon in a general setting. The main result shows that every linear value results from some least square approximation problem and that, conversely, every least square approximation problem with linear constraints yields a linear value.

This approach includes and extends previous results on so-called least square values and semivalues in the literature. In particular, is it demonstrated how known explicit formulas for solutions under additional assumptions easily follow from the general results presented here.
\end{abstract}

Keywords: cooperative game, least square approximation, least square value, pseudo-Boolean function, probabilistic value, semivalue, Shapley value MSC code: 91A12, 90C 20

${ }^{*}$ Mathematisches Institut, Universität zu Köln, Weyertal 80, 50931 Köln, Germany. Email: faigle@zpr.uni-koeln.de

$\dagger$ Corresponding author. Paris School of Economics, University of Paris I, 106-112, Bd. de l'Hôpital, 75013 Paris, France. Tel. (33) 144-07-82-85, Fax (33)-144-07-83-01. Email: michel.grabisch@univ-parisl.fr. The corresponding author thanks the Agence Nationale de la Recherche for financial support under contract ANR-13-BSHS1-0010. 


\section{Introduction}

Approximation of high-dimensional quantities or complicated functions by simpler functions with linear properties from low-dimensional spaces has countless applications in physics, economics, operations research etc. In these applications, the quality of the approximation is usually measured by the Gaussian principle of least squared error, which is also the guiding optimality criterion in the present investigation. Our study addresses a particular case of such an approximation context with many applications in different fields related to operations research, namely decision theory, game theory and the theory of pseudo-Boolean functions.

Where $N$ is a finite set with $n=|N|$ elements and collection $2^{N}$ of subsets, a set function $v: 2^{N} \rightarrow \mathbb{R}$ assigns to every subset of $N$ a real number, and is by definition of exponential complexity (in $n$ ). Identifying subsets of $N$ with their characteristic (incidence) vectors (and thus $2^{N}$ with $\{0,1\}^{n}$ ), a set function can be viewed as a so-called pseudo-Boolean function $f:\{0,1\}^{n} \rightarrow \mathbb{R}$ (cf. Hammer and Rudeanu [10]). Of particular interest are those set functions which vanish on the empty set, since they represent cooperative TU games with $N$ being the set of players and the quantities $v(S)$ expressing the benefit created by the cooperation of the members of $S \subseteq N$ (see, e.g., Peleg and Sudhölter [12]). Under the additional stipulation of monotonicity, i.e., the property that $v(S) \leq v(T)$ holds whenever $S \subseteq T$, one arrives at so-called capacities, which are a fundamental tool in the analysis of decision making under uncertainty ( $c f$. Schmeidler [15]) or relative to several criteria (Grabisch and Labreuche [6]).

Being of exponential complexity, a natural question is to try to approximate general set functions by simpler functions, the simplest being the additive set functions, which are completely determined by the value they take on the $n$ singleton sets $\{i\}$ and are thus of linear complexity (in $n$ ). In the field of pseudo-Boolean functions, the question has been addressed by Hammer and Holzman [8] with respect to linear and quadratic approximations, while approximation of degree $k$ was studied by Grabisch et al. [7]. In decision theory, linear approximation amounts to the approximation of a capacity $\mu$ by a probability measure $P$ (an additive capacity satisfying the additional constraint that $P(N)=1$ ).

In game theory, the approximation of a game $v$ by an additive game (equivalently by a (payoff) vector in $\mathbb{R}^{N}$ ) is related to the concept of value or solution of a game: given $v$, find $x \in \mathbb{R}^{N}$ such that $\sum_{i \in N} x_{i}=v(N)$ and the $x_{i}$ represent as faithfully as possible the contribution of the individual players $i$ in the total benefit $v(N)$. A very natural approach for a value is to define it as the best least square 
approximation of $v$, under the constraint $\sum_{i \in N} x_{i}=v(N)$, the approximation being possibly weighted. Such values are called least square values. An early and important contribution to this cooperative solution concept is due to Charnes et al. [2], who gave the general solution for the weighted approximation with nonnegative weights, and exhibited the well-known Shapley value [16] as a least square value. Ruiz et al. [13], for example, generalized this approach and derived further values from least square approximation.

The aim of this paper is to provide a general view on the set function approximation problem by placing it in the context of quadratic optimization and bringing well-known tools of convex analysis to bear on the problem. This approach not only generalizes existing results but also points to interesting connections and facts. Our formulation will remain general, although we will adopt most of the time the notation and ideas from cooperative game theory, due to the great interest in this field towards values and how to obtain them.

Our main result exhibits, roughly speaking, linear values and least square values for cooperative games to represent two sides of the same coin: we find that every least square problem under linear constraints yields a linear value and that every linear value arises as such a least square value (Section 3).

The paper is organized as follows. Section 2 describes the general problem of least square approximation and gives the fundamental result which will be used in the sequel (Theorem 2.1). Section 4 concentrates on least square values, and establishes explicit solution formulas under mild conditions on the weights used in the approximation. This model generalizes the approach to the Shapley value and to an optimization problem given in Ruiz et al. [13]. We remark that, interestingly, the weights do not necessarily have to be all positive in our model. Finally, we show in Section 5]how Weber's [18] so-called probabilistic values arise naturally in the present context.

\section{Least square approximations and linear operators}

We begin by reviewing some basic facts from convex optimization 1 . For integers $k, m \geq 1$, we denote by $\mathbb{R}^{k}$ the vector space of all $k$-dimensional (column) vectors and by $\mathbb{R}^{m \times k}$ the vector space of all $(m \times k)$-matrices $M=\left[m_{i j}\right]$ with coefficients $m_{i j}$. Generally, $M^{T}$ denotes the transpose of a matrix (or coefficient vector) $M$.

\footnotetext{
${ }^{1}$ see, e.g., Faigle et al. [4] or any other textbook for more details
} 
Recall that any positive definite $(k \times k)$-matrix $Q=\left[q_{i j}\right]$ defines an inner product via

$$
\langle x \mid y\rangle_{Q}=x^{T} Q y=\sum_{j=1}^{k} \sum_{i=1}^{k} q_{i j} x_{i} y_{j}
$$

with the associated $Q$-norm $\|x\|_{Q}=\sqrt{\langle x \mid x\rangle_{Q}}$ on $\mathbb{R}^{k}$. Note that the choice $Q=I$ of the identity matrix $I$ yields the usual euclidian norm $\|x\|=\|x\|_{Q}$.

Fix now a matrix $A^{m \times k}$, a linear map $b: \mathbb{R}^{k} \rightarrow \mathbb{R}^{m}$ as well as a linear map $c: \mathbb{R}^{k} \rightarrow \mathbb{R}^{k}$. For any $v \in \mathbb{R}^{k}$, denote by $\hat{v}=\hat{v}(A, b, c)$ the optimal solution of the quadratic minimization problem

$$
\min _{A x=b(v)}\|c(v)-x\|_{Q}^{2}
$$

So, if the system $A x=b(v)$ of linear equations has at least one solution, $\hat{v}$ is the (uniquely determined) best approximation of $c(v)$ in the solution space of $A x=b(v)$ in the norm $\|\cdot\|_{Q}$. The key observation in our analysis is:

Lemma 2.1 Assume that $A x=b(v)$ has a solution for every $v \in \mathbb{R}^{k}$ and that the map $c: \mathbb{R}^{k} \rightarrow \mathbb{R}^{k}$ is linear. Then $v \mapsto \hat{v}$ is a well-defined linear operator.

Proof. Problem (1) is equivalent to the quadratic optimization problem

$$
\min _{A x=b(v)} \frac{1}{2} x^{T} Q x-c(v)^{T} x .
$$

Given that $Q$ is positive definite, it is well-known that $x$ is the unique optimal solution for problem (2) if and only if there is a vector $y$ such that the associated Karush-Kuhn-Tucker (KKT) system

$$
\begin{aligned}
Q x+A^{T} y & =c(v) \\
A x & =b(v)
\end{aligned}
$$

is satisfied. Since $b$ and $c$ are linear functions in $v$, one immediately deduces from (3) that also the optimal solutions of (1) are linear functions in $v$.

Theorem 2.1 The operator $f: \mathbb{R}^{k} \rightarrow \mathbb{R}^{k}$ is linear if and only if there is a matrix $A \in \mathbb{R}^{m \times k}$, a linear function $b: \mathbb{R}^{k} \rightarrow \mathbb{R}^{m}$ and a linear function $c: \mathbb{R}^{k} \rightarrow \mathbb{R}^{k}$ such that $A x=b(v)$ is always solvable and $f(v)=\hat{v}$ holds. 
Proof. Lemma2.1 shows that the condition of the Theorem is sufficient for $f$ to be linear. Conversely, any $f(v)$ is obviously the optimal solution of the problem

$$
\min _{x \in \mathbb{R}^{k}}\|f(v)-x\|_{Q}^{2} .
$$

Hence the Theorem is satisfied with the choice $A=0$ and $b=0$, for example.

\section{Values of cooperative games}

Let $N$ be a set of players of finite cardinality $n=|N|$ and let $\mathcal{N}$ be the collection of non-empty subsets $S \subseteq N$. A cooperative $T U$ game is a function $v: \mathcal{N} \rightarrow \mathbb{R}$ (which is usually thought to be extended to all subsets of $N$ via $v(\emptyset)=0$ ). So the set $\mathcal{G}=\mathbb{R}^{\mathcal{N}}$ of all cooperative $\mathrm{TU}$ games on $N$ is a vector space and isomorphic to $\mathbb{R}^{k}$ with $k=|\mathcal{N}|=2^{n}-1$.

The additive (cooperative) games correspond to those members $x \in \mathbb{R}^{\mathcal{N}}$ that satisfy the homogeneous system of linear equations

$$
x(S)-\sum_{i \in S} x_{i}=0 \quad(S \in \mathcal{N})
$$

and one may be interested in the approximation of a game $v \in \mathcal{G}$ by an additive game with certain properties. More general approximations might be of interest. For example, the linear constraints

$$
\begin{aligned}
\sum_{i \in N} x_{i} & =v(N) \\
\sum_{S \in \mathcal{N}} x(S) & =\sum_{S \in \mathcal{N}} v(S)
\end{aligned}
$$

would stipulate an approximation of $v$ by a game that induces an efficient value (the first equality) and, furthermore, preserves the total sum of the $v(S)$ (second equality). Since the right-hand-side constraints are linear in $v$ Lemma 2.1 says that least square approximations of this type are linear in $v$.

A function $\Phi: \mathcal{G} \rightarrow \mathbb{R}^{N}$ is value for $\mathcal{G}$. It is straightforward, to view $\Phi(v)$ actually as an additive game that assigns the worth $\Phi_{S}(v)$ to the set $S$ of players by setting

$$
\Phi_{S}(v)=\sum_{i \in S} \Phi_{i}(v) \quad(S \in \mathcal{N}) .
$$


Conversely, every additive game $v$ arises from a parameter vector $\varphi \in \mathbb{R}^{N}$ so that

$$
v(S)=\sum_{i \in S} \varphi_{i} \quad(S \in \mathcal{N})
$$

Hence the space of additive games is isomorphic with $\mathbb{R}^{N}$. Consequently, Theorem 2.1 implies that the linear values are those which arise from least square approximation problems with linear constraints.

\section{Least square values}

We have seen that every linear value $\Phi: \mathcal{G} \rightarrow \mathbb{R}^{N}$ can be interpreted as arising from a least square approximation problem. Special cases of seemingly more general least square problems have received considerable attention in the literature and led to the concept of least square values and semivalues. Take, for example, the weighted least square problem

$$
\min _{x \in \mathbb{R}^{N}} \sum_{S \in \mathcal{N}} \alpha_{S}(v(S)-x(S))^{2} \quad \text { s.t. } \quad \sum_{i \in N} x_{i}=v(N),
$$

where we set $x(S)=\sum_{i \in S} x_{i}$. So (4) asks for the best ( $\alpha$-weighted) least square approximation of a game $v$ by an additive game $x$ under the additional efficiency constraint $x(N)=v(N)$.

This problem has a long history. Hammer and Holzman $([8])^{2}$ studied both the above version and the unconstrained version with equal weights $\left(\alpha_{S}=1 \forall S\right)$, and proved that the optimal solutions of the unconstrained version yield the Banzhaf value [1] (see also Section 5 below). More general versions of the unconstrained problem were solved by Grabisch et al. [7] with the approximation being relative to the space of $k$-additive games (i.e., games whose Möbius transform vanishes for subsets of size greater than $k$ )

In 1988, Charnes et al. [2] gave a solution for the case with the coefficients $\alpha_{S}$ being uniform (i.e., $\alpha_{S}=\alpha_{T}$ whenever $|S|=|T|$ ) and strictly positive. As a particular case, the Shapley value was shown to result from the coefficient choice

$$
\alpha_{S}=\alpha_{s}=\left(\begin{array}{c}
n-2 \\
s-1
\end{array}\right)=\frac{(n-2) !}{(s-1) !(n-1-s) !} \quad(s=|S|) .
$$

\footnotetext{
${ }^{2}$ later published in [9]

${ }^{3}$ see also Ding [3], and Marichal and Mathonet [11]
} 
REMARK. Ruiz et al. [13] state that problem (4) has a unique optimal solution for any choice of weights (see Theorem 3 there). In this generality, however, the statement is not correct as neither the existence nor the uniqueness can be guaranteed. So additional assumptions on the weights must be made.

We will first present a general framework for dealing with such situations and then illustrate it with the example of regular weight approximations and probabilistic values.

\subsection{Weighted approximation}

For the sake of generality, consider a general linear subspace $\mathcal{F} \subseteq \mathbb{R}^{\mathcal{N}}$ of dimension $k=\operatorname{dim} \mathcal{F}$, relative to which the approximation will be made.

Let $W=\left[w_{S T}\right] \in \mathbb{R}^{\mathcal{N} \times \mathcal{N}}$ be a given matrix of weights $w_{S T}$. Let $c: \mathbb{R}^{\mathcal{N}} \rightarrow \mathbb{R}^{\mathcal{N}}$ be a linear function and consider, for any game $v$, the optimization problem

$$
\min _{u \in \mathcal{F}}(v-u) W(v-u)^{T}+c(v-u)^{T} \quad \text { with } c=c(v),
$$

which is equivalent with

$$
\min _{u \in \mathcal{F}} u W u^{T}-\tilde{c} u^{T}
$$

where $\tilde{c} \in \mathbb{R}^{\mathcal{N}}$ has the components $\tilde{c}_{S}=c_{S}+2 \sum_{T} w_{S T} v_{T}$. A further simplification is possible by choosing a basis $B=\left\{b_{1}, \ldots, b_{k}\right\}$ for $\mathcal{F}$. With the identification

$$
x=\left(x_{1}, \ldots, x_{k}\right) \in \mathbb{R}^{k} \longleftrightarrow u=\sum_{i=1}^{k} x_{i} b_{i} \in \mathcal{F},
$$

problem (7) becomes

$$
\min _{x \in \mathbb{R}^{k}} \sum_{i=1}^{k} \sum_{j=1}^{k} q_{i j} x_{i} x_{j}-\sum_{i=1}^{k} \bar{c}_{i} x_{i}
$$

with the coefficients

$$
q_{i j}=\sum_{S} \sum_{T} w_{S T} b_{i}(S) b_{j}(T) \quad \text { and } \quad \bar{c}_{i}=\sum_{S} \tilde{c}_{S} b_{i}(S)
$$

Note that $\bar{c}: \mathbb{R}^{\mathcal{N}} \rightarrow \mathbb{R}^{k}$ is a linear function. 
Let $A \in \mathbb{R}^{m \times k}$ be a constraint matrix and $b: \mathbb{R}^{\mathcal{N}} \rightarrow \mathbb{R}^{m}$ a linear function such that $A x=b(v)$ has a solution for every $v \in \mathbb{R}^{\mathcal{N}}$. If $Q=\left[q_{i j}\right] \in \mathbb{R}^{k \times k}$ is positive definite, the problem

$$
\min _{x \in \mathbb{R}^{k}} \sum_{i=1}^{k} \sum_{j=1}^{k} q_{i j} x_{i} x_{j}-\sum_{i=1}^{k} \bar{c}_{i} x_{i} \quad \text { s.t. } \quad A x=b
$$

has a unique optimal solution $x^{*}$ which is linear in $v$ (Lemma2.1). So we obtain the linear value $v \mapsto \hat{v}$ with components

$$
\hat{v}_{j}=u_{\{j\}}^{*} \quad(j \in N), \text { where } \quad u^{*}=\sum_{i=1}^{k} x_{i}^{*} b_{i} \in \mathcal{F} .
$$

In the model (4), for example, $\mathcal{F}$ is the space $\mathcal{C}$ of all additive games and has dimension $n$. The matrix $W$ is diagonal with the diagonal elements $w_{S S}=\alpha_{S}$. If $\alpha_{S}>0$ holds for all $S$, then $W$ is positive definite and the linearity of the implied value $v \mapsto \hat{v}$ follows directly from Lemma 2.1 .

Otherwise, let us choose for $B$ the basis of unanimity games $\zeta_{i}, i \in N$, for $\mathcal{C}$, where

$$
\zeta_{i}(S)= \begin{cases}1 & \text { if } i \in S \\ 0 & \text { if } i \notin S .\end{cases}
$$

The associated matrix $Q=\left[q_{i j}\right]$ in model (4) has the coefficients

$$
q_{i j}=\sum_{S \in \mathcal{N}} \alpha_{S} \zeta_{i}(S) \zeta_{j}(S)=\sum_{S \ni\{i, j\}} \alpha_{S}
$$

For establishing a linear value, it suffices that $Q$ be positive definite, which is possible even when some of the $\alpha_{S}$ are negative (see Examples4.1 and 4.2 below).

\subsection{Regular weights}

While Lemma 2.1 guarantees the existence of linear values resulting from approximation, explicit formulas can be given under additional assumptions on the weights. Restricting ourselves to objectives of type

$$
\sum_{S \in \mathcal{N}} \alpha_{S}\left(v_{S}-u_{S}\right)^{2}+\sum_{S \in \mathcal{N}} c_{S} u_{S}
$$


we propose a simple framework that nevertheless includes all the cases treated in the literature so far. We say that the weights $\alpha_{S}$ are regular if the resulting matrix $Q$ has just two types of coefficients $q_{i j}$, i.e., if there are real numbers $p, q$ such that

$$
q_{i j}= \begin{cases}q & \text { if } i=j \\ p & \text { if } i \neq j\end{cases}
$$

Example 4.1 Assume that the weights $\alpha_{S}$ are uniform and set $\alpha(|S|)=\alpha_{S}$. Then formula (10) yields

$$
q_{i j}=\sum_{s=2}^{n}\left(\begin{array}{c}
n-2 \\
s-2
\end{array}\right) \alpha(s) \quad \text { and } \quad q_{i i}=\sum_{s=1}^{n}\left(\begin{array}{c}
n-1 \\
s-1
\end{array}\right) \alpha(s)
$$

holds for all $i \neq j$. So $Q=\left[q_{i j}\right]$ is regular.

Lemma 4.1 Let $Q=\left[q_{i i}\right] \in \mathbb{R}^{k \times k}$ be regular with $q=q_{i i}$ and $p=q_{i j}$ for $i \neq j$. Then $Q$ is positive definite if and only if $q>p \geq 0$.

Proof. For any $x \in \mathbb{R}^{k}$, we have after some algebra

$$
x^{T} Q x=(q-p) \sum_{i=1}^{k} x_{i}^{2}+p \bar{x}^{2}
$$

where $\bar{x}=\sum_{i=1}^{n} x_{i}$, which makes the claim of the Lemma obvious.

Note that our model allows for possibly negative uniform coefficients, as shown in the following example.

Example 4.2 Let $n=3$. We get $p=\alpha_{2}+\alpha_{3}$ and $q=\alpha_{1}+2 \alpha_{2}+\alpha_{3}$. Letting $\alpha>0$, the following vectors $\left(\alpha_{1}, \alpha_{2}, \alpha_{3}\right)$ lead to a positive definite matrix $Q$ :

$$
(0, \alpha, 0), \quad(\alpha, 0, \alpha), \quad(0, \alpha,-\alpha), \text { etc. }
$$

For the remainder of this section, let $Q \in \mathbb{R}^{N \times N}$ be a regular matrix with parameters $q>p \geq 0, c \in \mathbb{R}^{N}$ a vector and $g \in \mathbb{R}$ a scalar. Setting $\mathbf{1}^{T}=$ $(1,1, \ldots, 1)$, the optimization problem 


$$
\min _{x \in \mathbb{R}^{N}} x^{T} Q x-c^{T} x \quad \text { s.t. } \quad \mathbf{1}^{T} x=x(N)=g
$$

has a unique optimal solution $x^{*} \in \mathbb{R}^{N}$. Moreover, there is a unique scalar $z^{*} \in \mathbb{R}$ such that $\left(x^{*}, z^{*}\right)$ is the unique solution of the associated KKT-system

$$
\begin{aligned}
Q x-z \mathbf{1} & =c / 2 \\
\mathbf{1}^{T} x & =g .
\end{aligned}
$$

Verifying this KKT-system, the proof of the following explicit solution formulas is straightforward.

Theorem 4.1 If $Q$ is regular, the solution $\left(x^{*}, z^{*}\right)$ of the KKT-system (12) is:

$$
\begin{array}{ll}
z^{*}=(2(q+(n-1) p) g-C) / n & \left(\text { with } C=c \mathbf{1}^{T}=\sum_{i \in N} c_{i}\right) \\
x_{i}^{*}=\left(c_{i}+z^{*}-2 p g\right) /(2 q-2 p) & (i \in N) .
\end{array}
$$

If $Q$ is furthermore positive definite, then $x^{*}$ is an optimal solution for (11).

In the case of uniform weights $\alpha(s)$, the formulas in Theorem 4.1 yield the formulas derived by Charnes et al. [2] for problem (4). To demonstrate the scope of Theorem 4.1, let us look at the extremal problem 4 studied by Ruiz et al. [14]

$$
\min _{x \in \mathbb{R}^{N}} \sum_{S \subseteq N} m_{S} d(x, S)^{2} \text { s.t. } x(N)=v(N),
$$

where $m_{S}>0$ and

$$
d(x, S)=\frac{v(S)-x(S)}{|S|}-\frac{v(N \backslash S)-x(N \backslash S)}{n-|S|} .
$$

Letting $v^{*}(S)=v(N)-v(N \backslash S)$ and

$$
\bar{v}(S)=\frac{(n-|S|) v(S)+|S| v^{*}(S)}{n}
$$

(and thus $n \bar{v}(N)=v(N)$ ), we find that problem (13) becomes

$$
\min _{x \in \mathbb{R}^{N}} \sum_{S \subseteq N} \alpha_{S}(\bar{v}(S)-x(S))^{2} \text { s.t. } x(N)=n \bar{v}(N) .
$$

\footnotetext{
${ }^{4}$ see also Sun et al. [17] for similar problems
} 
with $\alpha_{S}=n^{2} m_{S}\left(|S|^{2}(n-|S|)^{2}\right)^{-1}$. Because $v \mapsto \bar{v}$ and $v \mapsto g(v)=n \bar{v}(N)$ are linear mappings, the optimal solutions of (13) yield an efficient linear value for any choice of parameters $m_{S}$ such that the associated matrix $Q$ is positive definite.

If furthermore the weights $m_{S}$ (and hence the $\alpha_{S}$ ) are uniform, $Q$ is regular and the optimal solution can be explicitly computed from the formulas of Theorem 4.1 .

\section{Probabilistic values}

Weber [18] introduced the idea of a probabilistic value arising as the expected marginal contribution of players relative to a probability distribution on the coalitions. For example, a semivalue is a probabilistic value relative to probabilities that are equal on coalitions of equal cardinality.

For our purposes, it suffices to think of the marginal contribution of an element $i \in N$ as a linear functional $\partial_{i}: \mathcal{G} \rightarrow \mathbb{R}$, where $\partial_{i}^{v}(S)$ is interpreted as the marginal contribution of $i \in N$ to the coalition $S \subseteq N$ relative to the characteristic function $v$.

Probabilistic values can be studied quite naturally in the context of weighted approximations. Indeed, let $p$ be an arbitrary probability distribution on $\mathcal{N}$. Then the expected marginal contribution of $i \in N$ relative to the game $v$ is

$$
E\left(\partial_{i}^{v}\right)=\sum_{S \subseteq N} \partial_{i}^{v}(S) p_{S} .
$$

Let $\mu_{i} \in \mathbb{R}$ be an estimate value for the marginal contribution of $i \in N$. Then the expected observed deviation from $\mu_{i}$ is

$$
\sigma\left(\mu_{i}\right)=\sqrt{\sum_{S \in \mathcal{N}} p_{S}\left(\partial_{i}^{v}(S)-\mu_{i}\right)^{2}} .
$$

A well-known fact in statistics says that the deviation function $\mu_{i} \mapsto \sigma\left(\mu_{i}\right)$ has the unique minimizer $\mu=E\left(\partial_{i}^{v}\right)$, which can also be immediately deduced from the KKT conditions for the least square problem

$$
\min _{\mu \in \mathbb{R}} \sum_{S \in \mathcal{N}} p_{S}\left(\partial_{i}^{v}(S)-\mu\right)^{2}
$$


The values of Shapley and Banzhaf. Shapley's [16] model assumes that player $i$ contributes to a coalition $S$ only if $i \in S$ holds and that, in this case, $i$ 's marginal contribution is evaluated as

$$
\partial_{i}^{v}(S)=v(S)-v(S \backslash i)
$$

So only coalitions in $\mathcal{N}_{i}=\{S \subseteq N \mid i \in S\}$ need to be considered. In order to speak about the "average marginal contribution", the model furthermore assumes:

(i) The cardinalities $|X|$ of the coalitions $X \in \mathcal{N}_{i}$ are distributed uniformly.

(ii) The coalitions $X \in \mathcal{N}_{i}$ of the same cardinality $|X|=s$ are distributed uniformly.

Under these probabilistic assumptions, the coalition $S \in \mathcal{N}_{i}$ of cardinality $|S|=s$ occurs with probability

$$
p_{S}=\frac{1}{n} \cdot \frac{1}{\left(\begin{array}{l}
n-1 \\
s-1
\end{array}\right)}=\frac{(s-1) !(n-s) !}{n !},
$$

which exhibits the Shapley value as a probabilistic (and hence approximation) value:

$$
\sum_{S \in \mathcal{N}_{i}} p_{S}[v(S)-v(S \backslash i)]=\sum_{S \in \mathcal{N}} p_{S}[v(S)-v(S \backslash i)]=\Phi_{i}^{\mathrm{Sh}}(v) .
$$

REMARK. Among the probabilistic values, the Shapley value can also be characterized as the one with the largest entropy (Faigle and Voss [5]).

In contrast to the Shapley model, the assumption that all coalitions in $\mathcal{N}_{i}$ are equally likely assigns to any coalition $S \in \mathcal{N}_{i}$ the probability

$$
\bar{p}_{S}=\frac{1}{2^{n-1}}
$$

with the Banzhaf value [1] as the associated probabilistic value:

$$
\sum_{S \in \mathcal{N}_{i}} \bar{p}_{S}[v(S)-v(S \backslash i)]=\sum_{S \in \mathcal{N}} \bar{p}_{S}[v(S)-v(S \backslash i)]=B_{i}^{v}
$$




\section{References}

[1] J.F. Banzhaf. Weighted voting does not work: A mathematical analysis. Rutgers Law Review, 19:317-343, 1965.

[2] A. Charnes, B. Golany, M. Keane, and J. Rousseau. Extremal principle solutions of games in characteristic function form: core, Chebychev and Shapley value generalizations. In J.K. Sengupta and G.K. Kadekodi, editors, Econometrics of Planning and Efficiency, pages 123-133. Kluwer Academic Publisher, 1988.

[3] G. Ding, R. Lax, J. Chen, P. Chen, and B. Marx. Transforms of pseudoboolean random variables. Discrete Applied Mathematics, 158:13-24, 2010.

[4] U. Faigle, W. Kern, and G. Still. Algorithmic Principles of Mathematical Programming. Springer, Dordrecht, 2002.

[5] U. Faigle and J. Voss. A system-theoretic model for cooperation, interaction and allocation. Discrete Applied Mathematics, 159:1736-1750, 2011.

[6] M. Grabisch and C. Labreuche. Fuzzy measures and integrals in MCDA. In J. Figueira, S. Greco, and M. Ehrgott, editors, Multiple Criteria Decision Analysis, pages 563-608. Kluwer Academic Publishers, 2005.

[7] M. Grabisch, J.-L. Marichal, and M. Roubens. Equivalent representations of set functions. Mathematics of Operations Research, 25(2):157-178, 2000.

[8] P. L. Hammer and R. Holzman. On approximations of pseudo-Boolean functions. RUTCOR Research Report RRR 29-87, State University of New Jersey, 1987.

[9] P. L. Hammer and R. Holzman. On approximations of pseudo-Boolean functions. ZOR - Methods and Models of Operations Research, 36:3-21, 1992.

[10] P. L. Hammer and S. Rudeanu. Boolean Methods in Operations Research and Related Areas. Springer, 1968.

[11] J.-L. Marichal and P. Mathonet. Weighted Banzhaf power and interaction indexes through weighted approximations of games. Eur. J. of Operations Research, 211:352-358, 2011. 
[12] B. Peleg and P. Sudhölter. Introduction to the theory of cooperative games. Kluwer Academic Publisher, 2003.

[13] L. M. Ruiz, F. Valenciano, and J. M. Zarzuelo. The family of leastsquare values for transferable utility games. Games and Economic Behavior, 24:109-130, 1998.

[14] L. M. Ruiz, F. Valenciano, and J. M. Zarzuelo. Some new results on least square values for tu games. TOP, 6:139-158, 1998.

[15] D. Schmeidler. Subjective Probability and Expected Utility without Additivity. Econometrica, 57(3):571-587, 1989.

[16] L. S. Shapley. A value for $n$-person games. In H. W. Kuhn and A. W. Tucker, editors, Contributions to the Theory of Games, Vol. II, number 28 in Annals of Mathematics Studies, pages 307-317. Princeton University Press, 1953.

[17] H. Sun, Z. Hao, and G. Xu. Optimal solutions for TU-games with decision approach. Preprint, Northwestern Polytechnical University, Xi' an, Shaanxi, China, 2013.

[18] R.J. Weber. Probabilistic values for games. In: A.E. Roth (ed.), The Shapley Value, Cambrigde University Press, Cambridge, 101-120, 1988. 